International Journal of Biology, Pharmacy and Allied Sciences (IJBPAS) 'A Bridge Betueen Caboratory and QRendo'

WwW.iibpas.com

\title{
REVIEW ON MICROSPONGE AS ADVANCED PARTICULATE DRUG DELIVERY SYSTEM
}

\section{SUJIT TAMBE*, VAIBHAV THOMBARE, SHANKAR DHOBALE, SURESH JADHAV AND DUSHYANT GAIKWAD}

Vishal Junnar Seva Mandal's Vishal Institute of Pharmaceutical Education and Research, Ale, Tal. Junnar, Dist. Pune, M.S., India

*Corresponding Author: E Mail: tambesujit05@gmail.com

Received 27 ${ }^{\text {th }}$ April 2021; Revised $25^{\text {th }}$ June 2021; Accepted $1^{\text {st }}$ Aug. 2021; Available online $1^{\text {st }}$ Oct. 2021

\section{https://doi.org/10.31032/IJBPAS/2021/10.10.1045}

\begin{abstract}
A number of advancements have been made in the medication delivery system in order to accomplish the aims of improved efficacy and cost effectiveness in therapy. The microsponge drug delivery system, which provides controlled release and site specific administration of active components, is one of the most recent, innovative, and rapidly emerging technologies. Microspheres with a size range of 5-300m that are strongly crosslinked, porous, and polymeric. Due to qualities such as reduced side effects, increased stability, better formulation flexibility, and enhanced product performance, this system is emerging as an useful choice for topical drug administration. It has a wide range of uses in the administration of oral, topical, ophthalmic, and biopharmaceuticals. Microsponge technology is described in depth, including formulation techniques, assessment, programmable release mechanisms, and applications.
\end{abstract}

Keywords: Microsponges; Controlled release; Quasi emulsion solvent diffusion; Programmable drug release; Oral administration; Topical drug delivery

\section{INTRODUCTION}

The skin was used as the entry portal for transdermal delivery systems class. The differential systems for systemic drugs in the scientists' biggest challenge right now is 
regulating the rate at which active pharmaceutical ingredients are delivered to a predetermined location. The goal of the researcher was to develop a precise controlled release drug delivery system that would improve patient safety and adherence [1]. Microsponges Delivery System (MDS) is highly cross-linked, porous, polymeric microspheres that could entrap a wide range of APIs and then release them into the skin over time and in reaction to a trigger. They range from 5-300 $\mu \mathrm{m}$ in diameter and a typical $25 \mu \mathrm{m}$ sphere can have up to 250,000 pores and an internal pore structure of equivalent to 10 feet in length, giving a total pore volume of approximately $1 \mathrm{ml} / \mathrm{g}$ for extensive retention of the drug. The surface can vary from 20 to $500 \mathrm{~m} / \mathrm{g}$ and 2 pore volumes of 0.1 to $0.3 \mathrm{~cm} / \mathrm{g}$. This creates a large reservoir within 3 microsponges, which can be loaded with active ingredients up to their own weight $[2,3,4]$.

Microsponges are porous microsphere-based polymeric delivery devices. They are spherical sponge-like particles with a porous surface. Furthermore, they may improve drug stability, minimize adverse effects, and positively alter drug release. Microsponge technology offers several advantages that make it a useful drug delivery mechanism. Microsponge Systems are made up of microscopic polymer-based microspheres that can suspend or entrap a wide range of components before being integrated into a manufactured product like a gel, cream, liquid, or powder. MDS can effectively boost the effectiveness of topically active drugs while also improving their safety, product stability, and cosmetic characteristics [5].

\section{History of Microsponge}

Microsponge technology was developed by Won in 1987 and the original patents were granted to Advanced Polymer Systems, Inc. This company has developed many variations of the procedures and these are applied to both cosmetics and over-the-counter (OTC) products and prescription medicines. Currently, this exciting technology is licensed to Cardinal Health, Inc., for use in topical products. Scanning electron microscopy of the microsponge particle reveals that the internal structure is the "bag of beads". The interstitial pores formed due to spaces between the beads and can entrap much wide range of active ingredients such as emollients, fragrances, essential oils, sunscreens, anti-infective and antiinflammatory agents $[6,7]$.

\section{Advantages of microsponge drug delivery systems: $[8,9]$ \\ - Enhanced product performance. \\ - Extended release.}


- Diminish irritation and hence enhanced patient

- Compliance.

- Improved product elegancy.

- Allows for novel product forms.

- Improves efficacy in treatment.

- Cure or control confirm more promptly.

- Improve control of condition.

- Improve bioavailability of same drugs

- Flexibility to develop novel product forms.

- Non-irritating, non-mutagenic, nonallergenic and non-toxic

- Improves thermal, physical and chemical stability

- Allows incorporation of immiscible products.

- Improves material processing e.g. liquid can be converted to powders

\section{Limitations: $[10,11]$}

The use of organic solvents has hazards such as toxicity and flammability. Traces of residual monomers in the bottom-up approach can be toxic and harmful to health. However, these limitations can be overcome by suitable measures for quality control in connection with the optimization and standardization of processes e.g. postmanufacturing washing.
Potential aspects of microsponge drug delivery system: [12-14]

- Shows acceptable stability over $\mathrm{pH}$ ranging from 1 to 11 and at high temperatures (up to $130^{\circ} \mathrm{C}$ ).

- Shows compatibility with various excipients and solvents.

- Entrapment efficiency found up to 50 to $60 \%$.

- Characterized by free flowing properties.

- They do not need sterilization or addition of preservatives because average pore size of microsponges is very small $(0.25 \mu \mathrm{m})$ to prevent the penetration of bacteria,

- Non-allergenic, non-irritating, nonmutagenic and non-toxic.

- Absorbs oil up to 6 times their weight without drying

Characteristics of active agents that is entrapped in microsponges: $[15,16]$

- Either fully miscible in monomer as well or capable of being made miscible by addition of small amount of awater immiscible solvent.

- Should not increase the viscosity of the mixture during formulation and inert to monomers.

- Water immiscible or nearly slightly soluble. 
- The microsponges' spherical structure should not collapse.

- In conditions of polymerization it should be stable in contact with polymerization catalyst.

- The vehicles will deplete the microsponges before the application, if the solubility of actives in the vehicle is not limited.

- Cosmetic problems can be avoided by using not more than 10 to $12 \% \mathrm{w} / \mathrm{w}$ microsponges incorporated into the vehicle.

- The payload and polymer design of the active microsponges must be adjusted for the desired release rate during a particular time period.

- Microsponge-entrapped ingredients can subsequently be used in a variety of products, including creams, gels, powders, lotions, and soaps.

Beneficial compared to other pharmaceutical formulations:

\section{A) Beneficial over conventional pharmaceutical formulations:}

As a topical drug delivery technique, semisolid or biphasic liquid systems are commonly used. They release the drug into the epidermis, which is the top layer of the skin. The drug was released quickly in these traditional formulations, and it may have absorbed and accumulated in the epidermis and dermis layers of the skin. The drug's high concentration causes adverse effects, inflammation, and toxicity. With the microsponge drug delivery method, these issues are no longer an issue. They deliver the medication to the skin in a slow and regulated manner $[\mathbf{1 7}, \mathbf{1 8}]$.

\section{B) Benefiacial over Micro and Nano- formulations:}

In terms of compatibility with a wide range of medicines, microsponges outperform microspheres, microencapsulation, niosomes, lipid nanoparticles, nanotubes, and liposomes etc.(benzoyl peroxide, oxybenzone, itraconazole, econazole, hydroquinone, capsaicin) due to controlled release easy formulation technique, stability and high capacity of drug loading [19, 20].

\section{Active moieties and polymers employed in}

\section{microsponges formulations}

The development of a microsponge'cage' is caused by the employment of several polymers in the manufacture of microsponges for topical application. Other excipients are required in the manufacture of stable and effective microsponge formulations, in addition to the polymers and active compounds. This active compounds are discussed in Table 1 with their ratio. 
Table 1: Active moieties and polymers employed in microsponges formulations

\begin{tabular}{|c|c|c|}
\hline Active compounds & Polymer \\
\hline Benzoyl peroxide & Ethyl cellulose & \\
\hline Dicyclomine & Eudragit S100 & \\
\hline Hydroxyzine HCl & Eudragit RS100 & \\
\hline Mometasone furoate & Eudragit RS100 & Ethyl cellulose \\
\hline Acyclovir sodium & Eudragit RS100 \\
\hline Diclofenac diethylamine & Eudragit RSPO \\
\hline \multicolumn{2}{|c|}{ Terbinafine } & Methods of preparation of microsponges
\end{tabular}

The solubility properties of the medication and polymer have a big role in deciding which encapsulation technique to use. Drug loading in microsponges can be accomplished in one of two ways: one-step or two-step, as mentioned in liquid-liquid suspension polymerization and quasiemulsion solvent diffusion methods, both of which are based on the bottom-up and topdown approach. There are different other methods of microsponge preparations which are discussed below.

\section{1) Liquid-liquid / Free radical suspension polymerization:}

Starting with monomer, this is a bottom-up method. Free radical suspension polymerization in an emulsified liquid-liquid system was used to make microsponges. The monomers are referred to as "monomer phase" or "dispersed Phase" In general, a solution is formed comprised of monomers and actives, which are immiscible with water. The immiscible liquid phase
Drug-Polymer Ratio $1: 1,1: 3,1: 5,1: 7,1: 9,1: 11,1: 13$

$1: 3,1: 6,1: 9,1: 12$

$1: 1,1: 2,1: 3,1: 4$

$1: 3,1: 5,1: 7,1: 9,1: 11,1: 13$

$1: 1,1: 2,1: 3,1: 4,1: 5,1: 6$

1:1.8, 1:4, 1:1

containing the dispensed (or dissolved)

monomer is defined as "Polymerization medium".

Another liquid may be introduced to the monomer to produce a pore network in addition to the monomers and polymerization medium. When this liquid, also known as a "monomer diluent" or "porogen," is introduced to the polymerization procedure, it produces polymeric beads with open, porous structures that resemble sponges under SEM, thus the term "microsponges". Temperature is maintained because it decides the rate of degradation, free radical formation and thus effect on rate of polymerization.

Polymerization is achieved by activating the monomers by catalysis, higher temperature, or irradiation once the suspension has been formed with distinct droplets of the required size. The polymerization procedure created 1000 spherical microsponge cages that were linked to resemble a grape bunch, constructing a network of interconnected reservoirs where the porogen is confined. Solid particles were collected from the suspension once the polymerization process 
was completed. For future usage, the particles were washed and dried [28-30].

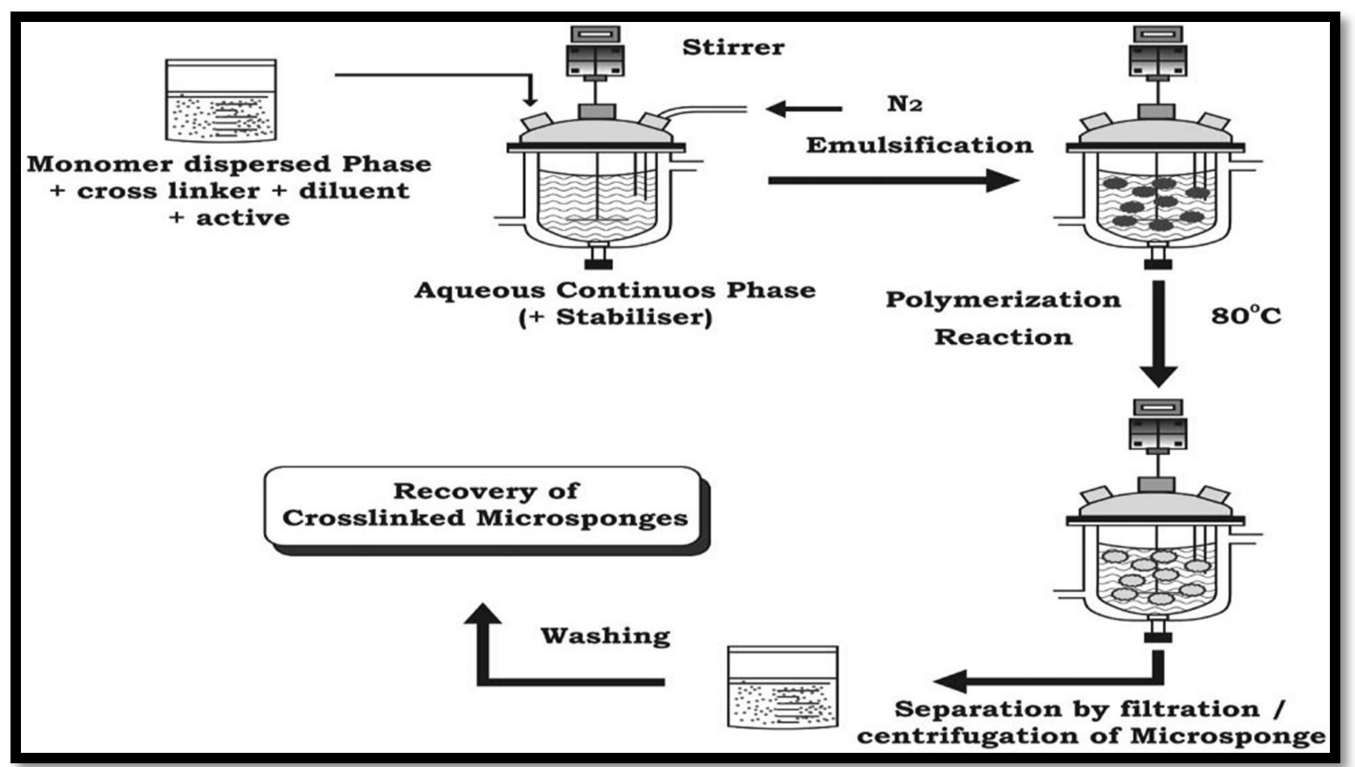

Figure 1: Liquid-liquid / Free radical suspension polymerization- system set up method

\section{2) Quasi-emulsion solvent diffusion method:}

Starting with preformed polymer, this is a top-down method. Similar to emulsions, this method involves the production of a quasiemulsion with two distinct phases: an internal phase and an external phase. The polymer is dissolved in ethyl alcohol in the internal organic phase, and the medication is dissolved in this solution by ultrasonication at room temperature. PVA solution in water makes up the external phase.

When you stir this emulsion, you get quasiemulsion globules, which are discrete emulsion globules. The solvent was then extracted from the globules, resulting in insoluble, rigid microparticles known as microsponges. The mixture was then filtered to separate the microsponges after enough stirring. After that, the microsponges were dried in an air oven. The finely dispersed droplets of the drug's polymeric solution (dispersed phase) solidify in the aqueous phase by counter diffusion of organic solvent and water out of and into the droplets, according to the theory.

The drug and polymer solubility was reduced by the diffused aqueous phase within the droplets, leading in co-precipitation of both components, and continuing diffusion of the organic phase resulted in additional solidification, resulting in matrix-type porous microspheres. In compared to the liquid-liquid suspension polymerization method, this approach offers the advantages of less drug 
exposure to ambient conditions and low solvent's solubility in aqueous medium or solvent residues in the result due to the volatile nature $[31,32]$.

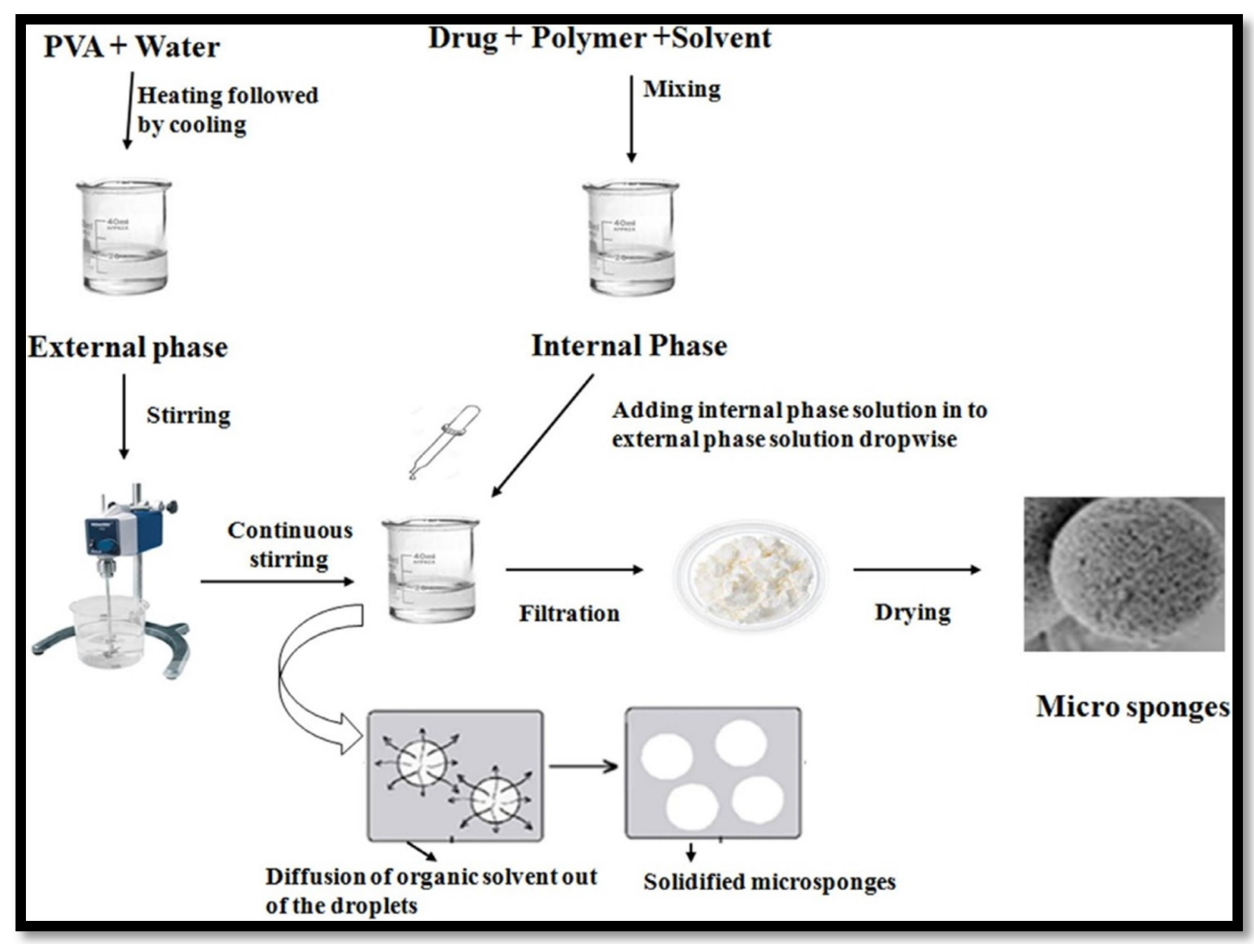

Figure 2: Quasi-emulsion solvent diffusion method set up

\section{3) Water in oil in water emulsion solvent diffusion:}

In this technique, an emulsifying agent such as span, polyethyleneimine, and stearyl amine was distributed in an organic polymeric solution via an internal aqueous phase. Following that, the w/o emulsion was dispersed in an external aqueous phase containing PVA to create a double emulsion. This technique captures both water-soluble and water-insoluble medicines. It can also be employed to hold thermolabile compounds, such as proteins, in place. Xanthan gum has also been described as an emulsifier to stabilise the internal w/o emulsion [33].

\section{4) Oil in Oil emulsion solvent diffusion:}

In contrast to the $\mathrm{w} / \mathrm{o} / \mathrm{w}$ technique, the oil in oil (o/o) emulsion was made with a volatile organic liquid as the internal phase, which was allowed to evaporate slowly and steadily while stirring was maintained. The volatile solvent in majority of the preparations is dichloromethane. The polymer employed is polyactide glycolic acid, with a span of 85 as the exterior phase. To make the microsponge, the internal phase was introduced to the dispersion medium dropwise with continuous stirring [34].

\section{5) Addition of porogen:}


Hydrogen peroxide or sodium bicarbonate as porogen used in internal phase which was dispersed in the polymeric solution to form a uniform dispersion system, and this was redispersed in aqueous phase containing PVA. The multiple emulsion was then given an initiator, and the organic solvent was allowed to evaporate, leaving behind the microparticles for making microsponges. Interconnected pores with diameters from ranges 5 to $20 \mu \mathrm{m}$ are formed as an effect of adding hydrogen peroxide [35].

\section{6) Lyophilisation:}

Microspheres were converted by gelatin method in this lyophilysation by quick removal of solvent leads to porous microspheres. Microspheres were incubated in the solution of chitosan hydrochloride and then lyophilized in this method. This quick and rapid method have disadvantages of producing cracked or shrunken microparticles due to quick elimination of solvent [36].

\section{7) Vibrating Orifice Aerosol Generator Method (VOAG):}

Firstly the preparation of lipid bilayered mesoporous silica particles required a vibrating orifice aerosol generator (VOAG). In method porous particles are synthesized by evaporation-driven surfactant templating in microdroplets by a VOAG method.
Tetraethylorthosilicate, ethanol, water and dilute hydrochloric acid were refluxed to prepare stock solution for the preparation of core particle. The stock solution was diluted with surfactant-containing solvent and agitated to allow monodisperse droplets to form using VOAG.The microspheres that were created were encapsulated in liposomes. These encapsulated particles can be used to deliver active ingredients to specific locations [37].

\section{8) Ultrasound-Assisted Production:}

This method is modified version of the liquid-liquid suspension polymerization. Monomer beta-cyclodextrin (BCD) and cross-linking agent diphenyl carbonate is used to synthesize the microsponges. Heating and sonication of the reaction mixture controls the size of microsponges. Then reaction mixture was cooled, the product obtained was milled to give rough particles that were washed with distilled water and then by ethanol. Cross-linked $\beta-\mathrm{CD}$ porous microparticles serve as carrier for efficient loading of drugs although entrapment of residues of the cross-linking agents that can be potentially toxic [38].

\section{9) Electrohydrodynamic Atomization Method:}

This approach resulted in chitosan porous microspheres. The chitosan solution was 
sonicated to produce bubbles, and the resulting bubble suspension was drawn into a syringe, perfused via a steel capillary using a syringe pump, and electrohydrodynamically atomized. The capillary's diameter was selected to ensure that all bubbles in the suspension were retained as it passed through it. The voltage utilised in the tests is completely determined by the chitosan content in the solution. A $4 \% \mathrm{w} / \mathrm{v}$ sodium hydroxide aqueous solution was used to cross-link the chitosan microspheres [39].

\section{Recent developments in microsponge drug delivery system}

Some researchers, pharmaceutical companies are developing advanced formulations. They are as follows and having more stability than microsponges.

\section{1) Nanosponges:}

This has been shown to be a good carrier for gas distribution. When cytotoxic is combined with a nanosponges carrier system, the drug's effectiveness is increased, allowing it to be utilised to target cancer cells. Crosslinking the $\beta-C D$ molecule with biphenyl carbonate produces $\beta-C D$ nanosponges. They can be utilised to make both hydrophilic and hydrophobic medicines.

\section{2) Nanoferrosponges:}

Nanoferrosponges are self-promising carriers with enhanced penetration towards a specific location due to an external magnetic reaction that allows carriers to penetrate deeper tissue before magnetic material is excluded, leaving a porous system behind.

\section{3) Porous Microbeads:}

Microbeads with a large number of pores are created by improving the properties of porous microspheres. Solid porous microbeads are made using polymerization and cross-linking techniques. Topical, buccal, and oral medication delivery systems employ these microbeads [40].

\section{CONCLUSION}

The Microsponge Delivery System can entrap a wide spectrum of actives and then release them onto the skin in a controlled and timed manner. It's a one-of-a-kind technique that uses microporous beads filled with active agents to administer topical drugs in a regulated manner. It may also be used for oral and biopharmaceutical drug delivery. The active component in a microsponge delivery system can be released on a timer or in reaction to other inputs. As a result, microsponge has a huge amount of potential and is a relatively new subject that needs to be investigated.

\section{REFERENCES}

[1] Jagtap S.C., Karale A.A., Ambekar A.W: Microsponge: A novel topical drug 
delivery system. Journal of Drug

Delivery Research (2014);3(4):1-9.

[2] Nacht $S$ and Kantz M. The Microsponges, A Novel Topical Programmable Delivery System. Chapter 15, In, Topical Drug Delivery System. Edited by David WO and Anfon HA, 42, 1992, 299-325.

[3] Namrata Jadhav, Vruti Patel, Siddhesh Mungekar, Manisha Karpe, Vilasrao Kadam, Microsponge delivery system: anupdated review, current status and future prospects, Journal of Scientific and Innovative Research2013; 2(6), 1097-1110.

[4] Embil K.,Nacht S. The Microsponge Delivery System (MDS): A topical delivery system with reduced irritancy incorporating multiple triggering mechanisms for the release of actives. J. Microencapsul.1996; 3(5), 575-588.

[5] Shivani Nanda, Mandeep Kaur, Nikhil Sood, Sahil Nagpal, Microsponge drug delivery system: an overview, World Journal of Pharmacy and Pharmaceutical Sciences,2013; 2(3), 1032-1043.

[6] Won R: Method for delivering an active ingredient by controlled time release utilizing a novel delivery vehicle which can be prepared by a process utilizing the active ingredients as a Porogen. 1987; US Patent No. 4690825

[7] Pawar AP, Gholap AP, Kuchekar AB, Bothiraja $\mathrm{C}$ and Mali AJ: Formulation and evaluation of optimized oxybenzone microsponge gel for topical delivery. Journal of Drug Delivery 2015; 15: 26168.

[8] Patidar K, Soni M, Saxena C, Soni P, Sharma DK. Microspongea versatile vesicular approach for transdermal drug delivery system. J Global Pharm Tec, 2(3), 2010, 154-164.

[9] N.H. Aloorkar, A.S. Kulkarni, D.J. Ingale and R.A. Patil, Microsponges as Innovative Drug Delivery Systems, International Journal of pharmaceutical Sciences and Nonotechnology, 2012; 5(1):143-151..

[10] Shyam S. M., Vedavathi T. Novel approach: microsponge drug delivery system. Int. J. Pharm. Sci.Res.2012; 3(4): 967-980.

[11] Srivastava R, Pathak K. Microsponges: a futuristic approach for oral drug delivery. Expert Opin. Drug Deliv.2012; 9(7): 863-878.

[12] Hibah Aldawsari and Shaimaa M. BadrEldin, Microsponges as promising vehicle for drug delivery and targeting: Preparation, characterization and applications, African Journal of Pharmacy and Pharmacology, 3(1), 2001, 873-881.

[13] Jain V, Singh R. Design and characterization of colon-specific drug delivery system containing paracetamol 
microsponges. Arch. Pharm. Res. 34, 2011, 733-740.

[14] Vyas SP, Khar RK. Targeted and controlled drug delivery: novel carrier systems. CBS Publications, 1st ed., New Delhi, 453, 2002.

[15] Swetha, Gopal Rao, Venkata Ramana K, Niyaz Basha B and Koti Reddy V. Formulation and in-vitro evaluation of etodolac entrapped in microsponge based drug delivery system. Int $\mathbf{J}$ Pharma, 1(2), 2011, 73-80.

[16] Kaity, S, Maiti, S, Ghosh, A.K, Pal, D, Ghosh, A., \& Banerjee, S: Microsponges: A novel strategy for drug delivery system. Journal of Advanced Pharmaceutical Technology \& Research (2010);1(3):283-290.

[17] Patel UB, Shah $\mathrm{CN}$ and Patel HM: Formulation and development of aceclofenac loaded microsponge for topical delivery using quality by design approach. International Journal of Advanced Pharmaceutics 2018; 7(4): 17-32.

[18] Sonali and Rahul: Formulation and evaluation of prednisolone loaded microsponges for colon drug delivery: In-vitro and pharmacokinetic study. International Journal of Pharmaceutical Sciences and Research 2014; 5(5): 1994-05.

[19] Lengyel M, Kállai-Szabó N, Antal V, Laki AJ, Antal I. Microparticles,
Microspheres, and Microcapsules for Advanced Drug Delivery. Scientia Pharmaceutica. 2019; 87(3):20.

[20] Mohite P.B, Khanage S.G., Harishchandre S.S, Shirsath Yogita. Recent advances in microsponges drug delivery system. JCR. 2016; 3(1): 9-16.

[21] Nokhodchi A, Jelvehgari M, Siahi MR, Mozafari MR. Factorsaffecting the morphology of benzoyl peroxidemicrosponges. Micron 2007;38(8):834-40

[22] Jain V, Singh R. Dicyclomine-loaded Eudragit ${ }^{\circledR}$-basedmicrosponge with potential for colonic delivery:preparation and characterization. Trop J Pharm Res2010;9:67-72.

[23] Zaki Rizkalla CM, Latif Aziz R, Soliman II. In vitro andin vivo evaluation of hydroxyzine hydrochloridemicrosponges for topical delivery. AAPS Pharm. Sci. Tech., 2011;12:989-1001.

[24] Rekha U, Manjula BP. Formulation and evaluation of microsponges for topical drug delivery of mometasonefuroate. Int J Pharm Pharm Sci 2011; 3(4): 1337.

[25] Chandramouli Y, Firoz S, Rajalakshmi R, Vikram A,Yasmeen BR, Chakravarthi RN. Preparation and evaluationof microsponge loaded controlled release topical gel 
ofacyclovir sodium. Int $\mathrm{J}$ Biopharm 2012;3(2):96-102.

[26] Osmani RA, Aloorkar NH, Ingale DJ, Kulkarni PK, Hani U,Bhosale RR, et al. Microsponges based novel drug delivery system for augmented arthritis therapy. Saudi Pharm J2015;23:562-72.

[27] Barde PM, Basarkar GD. Formulation, development and invitro evaluation of terbinafine $\mathrm{HCl}$ microsponge gel. Int $\mathrm{J}$ Pharm Sci Rev Res 2015;32:310-14.

[28] Chadawar V., Shaji J. Microsponge Delivery System. Current Drug Delivery. 2007 ; 4: 123-129.

[29] Aritomi H., Yamasaki Y., Yamada K., Honda H, Koshi M. Development of sustained release formulation of chlorpheniramine maleate using powder coated microsponges prepared by dry impact blending method. Journal of Pharmaceutical Sciences and Technology. 1996; 56(1): 49-56.

[30] Rajurkar VG, Tambe $\mathrm{AB}$ and Deshmukh VK: Topical antiinflammatory gels of naproxen entrapped in eudragit based microsponge delivery system. Journal of Advanced Chemical Engineering 2015; 5(2): 2-6

[31] Kawashima $Y$, Niwa $T$, Takeuchi $H$, Hino T, Ito Y. Control of Prolonged Drug Release and Compression Properties of Ibuprofen Microsponges with Acrylic Polymer, Eudragit RS, by
Changing Their Intraparticle Porosity. Chemical and pharmaceutical bulletin. 1992; 40(1):196-201.

[32] Vyas L.K., Tapar K.K., Laddha B.H., Lahoti A.O., Nema R.K. Formulation and development of anti-blemish preparation using microsponge technology. J. Chem. Pharm. Res. 2010; 2(5):562-571.

[33] Maiti S, Kaity S, Ray S, et al. Development and evaluation of xanthan gum-facilitated ethyl cellulose microsponges for controlled percutaneous delivery of diclofenac sodium. Acta Pharm. 2011; (61): 25770.

[34] Zaki Rizkalla CM, Latif Aziz R, Soliman II. In vitro and in vivo evaluation of hydroxyzine hydrochloride microsponges for topical delivery. AAPS Pharm. Sci. Tech. 2011;12(3):989-1001.

[35] Bae SE, Son JS, Park K, et al. Fabrication of covered porous PLG A microspheres using hydrogen peroxide for controlled drug delivery and regenerative medicine. $\mathrm{J}$ Control Release 2009; (133):37-43.

[36] Liu LS, Liu SQ, Ng SY, et al. Controlled release of interleukin-2 for tumour immunotherapy using alginate/chitosan porousmicrospheres. J Control Release 1997;(43):65-74 
[37] Lopez GP, Buranda T, Gopalaraju VRR, et al. Biologically functionalized porous microspheres. US2004005352; 2004.

[38] Shende P, Pawar S, Trotta F, Diversity of $\beta$ - cyclodextrin based nanosponges for transformation of actives, International Journal of Pharmaceutics, $2019 ; 565: 333-350$.

[39] Pancholi K, Ahras N, Stride E, Edirisinghe M, Novel electrohydrodynamic preparation of porous chitosan particles for drug delivery, Journal of Material Science: Mater Med, 2009; 20:917-923.

[40] Kar AK, Kar B, Parya H, Kundu S, Hirawat R, A novel approach on microsponge: multifunctional dosage form, International Journal of Pharmaceutical Sciences Review and Research,2018; 51(2):64-72. 